ARTICLE

Received 5 Feb 2015 | Accepted 13 Jul 2015 | Published 2 Sep 2015

\title{
Signature of magnetic-dependent gapless odd frequency states at superconductor/ferromagnet interfaces
}

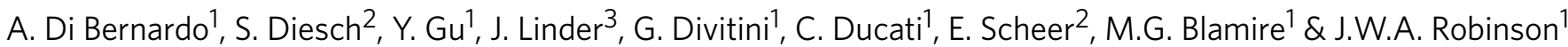

The theory of superconductivity developed by Bardeen, Cooper and Schrieffer (BCS) explains the stabilization of electron pairs into a spin-singlet, even frequency, state by the formation of an energy gap within which the density of states is zero. At a superconductor interface with an inhomogeneous ferromagnet, a gapless odd frequency superconducting state is predicted, in which the Cooper pairs are in a spin-triplet state. Although indirect evidence for such a state has been obtained, the gap structure and pairing symmetry have not so far been determined. Here we report scanning tunnelling spectroscopy of $\mathrm{Nb}$ superconducting films proximity coupled to epitaxial $\mathrm{Ho}$. These measurements reveal pronounced changes to the $\mathrm{Nb}$ subgap superconducting density of states on driving the Ho through a metamagnetic transition from a helical antiferromagnetic to a homogeneous ferromagnetic state for which a BCS-like gap is recovered. The results prove odd frequency spin-triplet superconductivity at superconductor/inhomogeneous magnet interfaces.

\footnotetext{
${ }^{1}$ Department of Material Science and Metallurgy, University of Cambridge, 27 Charles Babbage Road, Cambridge CB3 OFS, UK. ${ }^{2}$ Department of Physics, University of Konstanz, Konstanz 78457, Germany. ${ }^{3}$ Department of Physics, Norwegian University of Science and Technology, Trondheim N-7491, Norway. Correspondence and requests for materials should be addressed to J.W.A.R. (email: jjr33@cam.ac.uk)
} 
n 1961, Ivar Giaever reported ${ }^{1}$ the energy gap in the excitation spectrum of the underlying metallic band structure of superconducting $\mathrm{Al}$, which directly verified the theory of superconductivity developed by Bardeen, Cooper and Schrieffer ${ }^{2}$ (BCS). The superconducting density of states (DOS) is determined by the symmetry of the Cooper pair wavefunction in time, orbital and spin spaces. For BCS superconductors such as $\mathrm{Al}$ and $\mathrm{Nb}$, the Cooper pairs are in a spin-singlet state in which their wavefunction is even with respect to exchange of time coordinates-this is so-called even-frequency superconductivity.

At a superconductor/ferromagnet (S/F) interface, in which the magnetization of the $\mathrm{F}$ layer is inhomogeneous, an additional odd frequency pairing symmetry is predicted in which the Cooper pairs are in a spin-triplet state with either zero $\left(S_{z}=0\right)$ or a net spin projection ${ }^{3,4}\left(S_{z}= \pm 1\right)$. For simplicity, we will hereafter refer to these different types of Cooper pairs as spin-zero and spin-one states. The classification odd frequency stems from the fact that the wavefunction of the Cooper pairs is odd with respect to an exchange of the time coordinates.

Indirect evidence for the generation of odd frequency triplet states has emerged from measurements of S/F/S Josephson junctions, which demonstrate a coherence length in $\mathrm{F}$ that is much longer than predicted for BCS (singlet) proximity effects $^{5-11}$ or in junctions with ferromagnetic insulators with high spin-polarizations ${ }^{12}$. Odd frequency triplet pairing has also been inferred from critical temperature measurements of $\mathrm{S} / \mathrm{F} / \mathrm{F}$ spin valves $^{13-19}$. The superconducting excitation spectrum for the odd frequency triplet state should be gapless, meaning the net subgap DOS should be enhanced rather than suppressed ${ }^{20,21}$ at certain energies (voltages). To date, tunnelling conductance measurements to detect odd frequency superconductivity have mainly focused on the F-side of metallic ${ }^{22-24}$ and oxide ${ }^{25-27} \mathrm{~S} / \mathrm{F}$ interfaces for which a superconducting minigap appears in the DOS because of the superconductor proximity effect $^{28}$, and which has been predicted to contain both even and odd frequency components and to disappear on a length scale of the ferromagnet singlet pair coherence length $\xi_{\mathrm{F} \text {,Singlet }}$ of 1-5 nm. However, discriminating between the odd and even frequency components in the DOS has proven to be controversial and so the results have so far been inconclusive. Eschrig and Löfwander theoretically demonstrated that a spin-active interface also enhances odd frequency pairing correlations on the S-side of an $\mathrm{S} / \mathrm{F}$ interface ${ }^{29}$. Here the superconducting DOS should in principle be easier to detect because, unlike on the F-side, the DOS is not masked by the normal state background.
To isolate the odd frequency contributions with certainty, it is necessary to amplify them. This can be achieved by introducing a spin-active layer - a magnetic layer with a magnetization direction that is non-parallel to the F layer-at the S/F interface ${ }^{3}$. Such a layer can enhance the pairing amplitude of the spin-triplet state. The mechanism behind this effect first involves mixing the singlet pairs because of the spin-dependent phases picked up by electron scattering at the $\mathrm{S} / \mathrm{F}$ interface, which then results in the emergence of a spin-zero triplet state. The presence of a spinactive layer then rotates the triplet pairs in spin-space and spin-aligned triplet pairs form.

In this article we report scanning tunnelling DOS measurements on superconducting films of $\mathrm{Au} / \mathrm{Nb}$ proximity coupled to an epitaxial film of Ho. These measurements reveal pronounced changes to the subgap DOS in $\mathrm{Nb}$ that sensitively depend on the magnetic phase of Ho; by controlling the magnetic structure of Ho we are able to distinguish between singlet and triplet pair correlations in the superconducting DOS of Nb, since through the magnetic phase of Ho we are able to amplify (suppress) the triplet (singlet) components.

\section{Results}

Tuneable magnetism. To obtain discriminating evidence for the symmetry of the superconducting state at S/F interfaces and to correlate subgap structure to magnetism, we performed scanning tunnelling spectroscopy measurements on epitaxial $\mathrm{Nb}(20 \mathrm{~nm}) / \mathrm{Ho}(9.5 \mathrm{~nm}) / \mathrm{Nb}(6.5 \mathrm{~nm})$ thin films capped with a 3-nm-thick protective layer of $\mathrm{Au}$ (see Fig. 1a and for supporting $\mathrm{X}$-ray diffraction data see Supplementary Figs 1 and 2). The Au protects the structure from oxidation, while the 6.5-nm-thick non-superconducting base layer of $\mathrm{Nb}$ provides the correct seed for epitaxial growth ${ }^{30}$. The $\mathrm{Nb}(20 \mathrm{~nm}) / \mathrm{Ho}(9.5 \mathrm{~nm}) / \mathrm{Nb}(6.5 \mathrm{~nm})$ multilayer had a superconducting transition of $\sim 6.6 \mathrm{~K}$ and without Ho of $\sim 8.6 \mathrm{~K}$ (see Supplementary Fig. 3).

In single crystal Ho a basal plane helical magnetic phase forms below $133 \mathrm{~K}$ (refs 31,32), where the helix turn angle, the angle between magnetic moments in adjacent layers, is $30^{\circ}$. On application of a sufficiently large field parallel to the basal plane, a metamagnetic transition to a stable ferromagnetic (F) state takes place in which the moments are aligned in plane ${ }^{33}$. We show below and in ref. 30 that our Ho thin films behave in a similar way.

In Fig. $1 \mathrm{~b}$ we have plotted the in-plane magnetization versus field loop $M(H)$ for an epitaxial sample at $10 \mathrm{~K}$. The initial magnetization curve shows a transition to a square $\mathrm{F}$ hysteresis loop, which is stable over subsequent field cycles. The fact that
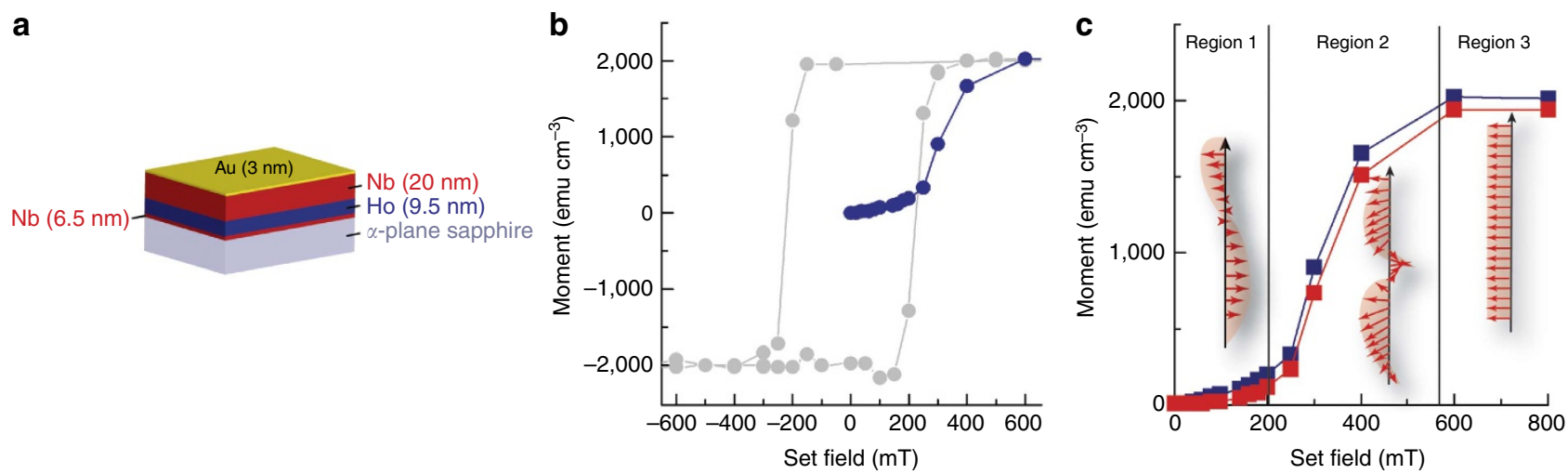

Figure 1 | Magnetic properties of the $\mathbf{A u} / \mathbf{N b} / \mathbf{H o} / \mathbf{N b}$ multilayer thin films. (a) Illustration of the sample structure. (b) Initial magnetization curve (blue) and $M(H)$ loop (grey) at $10 \mathrm{~K}$. Lines are a guide to the eye. (c) Magnetization at zero field $\left(M_{r} ;\right.$ red) and with the set field $H_{\mathrm{SET}}$ Switched on (blue). Lines are guides to the eye. Vertical (black) lines separate different magnetic phases of Ho: a bulk helix (region 1), coexisting helix and $\mathrm{F}$ component (region 2 ), and $\mathrm{F}$ state (region 3). 
the initial magnetization curve goes outside the loop demonstrates a phase transition to an $\mathrm{F}$ state. The saturation magnetization $\left(M_{\mathrm{s}}\right)$ reaches $\sim 2,026 \mathrm{emu} \mathrm{cm}^{-3}$, which is close to the theoretical value of $10.34 \mu_{\mathrm{B}}$ per atom ${ }^{34}$ and shows that the Ho has near bulk properties.

In Fig. 1c we have plotted the zero-field magnetic phase diagram of our Ho. This was created by the following procedure. First, an in-plane magnetic field (which we call the 'set field' or $\left.H_{\mathrm{SET}}\right)$ was applied and the corresponding magnetization was measured $\left(M\left(H_{\mathrm{SET}}\right)\right)$. This field was then switched off; therefore, the remanent magnetization $M_{\mathrm{r}}$ due to $H_{\mathrm{SET}}$ can be measured. This procedure was repeated with successively larger values of $H_{\text {SET }}$ until saturation was achieved. Below $H_{\text {SET }}=200-250 \mathrm{mT}$, $M_{\mathrm{r}}$ and $M\left(H_{\mathrm{SET}}\right)$ barely increase, which implies the helix phase in Ho is robust (region 1 of Fig. 1c), but beyond this field range, $M_{\mathrm{r}}$ and $M\left(H_{\mathrm{SET}}\right)$ sharply increase indicating the onset of the metamagnetic transition in which the helix deforms and contains a significant $\mathrm{F}$ component (region 2 of Fig. 1c). Beyond approximately $500 \mathrm{mT}$, the Ho is $\mathrm{F}$ (region 3 of Fig. 1c).

Scanning tunnelling spectroscopy was performed at $290 \mathrm{mK}$ with an IrPt tip (well below the superconducting transition temperature of the films investigated) mounted on a ${ }^{3} \mathrm{He}$ cryostat in which in-plane magnetic fields of up to $500 \mathrm{mT}$ could be applied ${ }^{35}$. The spatial distribution of the superconductingrelated spectral features was investigated and, in addition to BCS gaps, two different subgap structures consistently appeared in the DOS in the as-cooled tunnelling conductance $\mathrm{d} I / d V$ versus bias voltage $V$ as shown in Figs 2 and 3; $d I / d V$ is normalized to the normal state conductance at high bias $(5 \mathrm{mV})$. The most common non-BCS subgap structure consisted of a double peak in $\mathrm{d} I / d V$ at symmetrical values of bias voltage (which we name the double peak spectra; see Figs $2 \mathrm{a}$ and $3 \mathrm{e}$ ). In other areas, a conductance peak at zero-bias voltage was obtained (which we name the zero peak spectra; see Figs $2 \mathrm{~b}$ and $3 \mathrm{f}$ ); however, this feature was far less common (in $\sim 10 \%$ of scans).

Subgap dependence on film surface and magnetic phase. To rule out the possibility of spurious effects from surface defects as an explanation of the subgap structure and to ensure that the subgap structure is related to the superconductor proximity effect, we measured the spatial dependence of the tunnelling DOS and searched for correlations between the spectral features and the surface topography (Fig. 3): as shown, the subgap structure is sensitive to the surface topography and no subgap structure was obtained in regions with a high concentration of defects, showing up in the topography map as increased noise or protrusions and as abundance of black areas in the spectral map (see, for example, around $x=-20 \mathrm{~nm}$ and $y=-40 \mathrm{~nm}$ in
Fig. 3a and b). Around grain boundary-like regions we observed either BCS-like gaps (often shallow) or metallic structure, but on defects we only observed noisy metallic spectra. For further details on how the spectral map in Fig. 3b is obtained from the measured DOS spectra, see Supplementary Fig. 4 and Supplementary Note 1.

Conductance maps at different voltages $(0,-0.35,-0.5$ and $-1.5 \mathrm{mV}$ ) were also determined from the $16 \times 16$ matrix of DOS spectra recorded on the sample area shown in Fig. 3 (see Supplementary Figs 5 and 6). These maps show a good reproducibility of the local spectral features (Fig. 3c-f), which is in agreement with the spectral map reported in Fig. $3 \mathrm{~b}$.

We attribute the BCS-like spectra to positions where the Ho magnetic structure is disturbed such that no pronounced noncollinearity of the magnetization is present. While we cannot confirm this, to be certain that the BCS gap structure is not related to discontinuities of Ho and therefore to the absence of a magnetic proximity effect, we performed transmission electron microscopy (TEM) on cross-sectional lamellae of the specimen prepared by focused ion beam (FIB); see Fig. 4 and Methods for further details. Low-magnification scanning transmission electron microscopy images (Fig. 4a,b) show that the Ho layer is continuous, which is further supported by energy-dispersed X-ray data (see Fig. 4c-e). High-resolution TEM (Fig. 4f-i) shows that the $\mathrm{Nb}$ and Ho layers are crystalline, with little evidence of interdiffusion. Note that during the fabrication of the TEM lamella the top half of the $\mathrm{Nb}$ layer and the entire Au layer are damaged and amorphized because of a combination of $\mathrm{Ga}^{+}$ion implantation occurring during the FIB processing and the deposition of a $\mathrm{Pt} / \mathrm{C}$ capping layer.

The metallic spectra (Fig. 3c) might indicate surface contamination that suppresses superconductivity locally or that disables proper phase locking of the lock-in measurement. Importantly, the subgap structures (Fig. 2) do not appear preferentially at defects, but are more pronounced in clean regions, which is consistent with the presence of a stronger superconductor proximity effect.

Finally, we also tested control samples of $\mathrm{Au}(3 \mathrm{~nm})$ on epitaxial $\mathrm{Nb}(20 \mathrm{~nm})$ without Ho. On these samples only BCS gaps or metallic structure were ever observed (see Supplementary Fig. 7), which confirms that the subgap structure observed in Figs 2 and 3 are related to an unconventional proximity effect due to Ho interacting with $\mathrm{Nb}$.

The most striking evidence that the subgap structure is related to the interaction of $\mathrm{Ho}$ with $\mathrm{Nb}$ and therefore to an exotic proximity effect was obtained by monitoring the dependence of the subgap features to the magnetic structure of Ho by applying magnetic fields. We first focus on the effect that magnetic
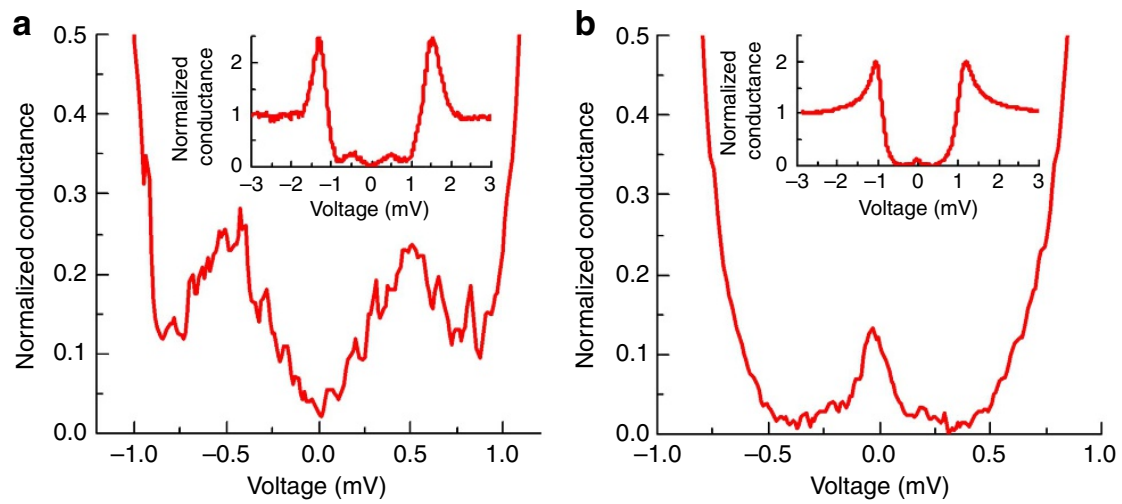

Figure 2 I Zero-field superconducting subgap structure. Typical tunnelling conductance dl/dV versus bias voltage $V$ spectra acquired at 290 mK determined on two different surface regions showing double peak (a) or zero peak (b) enhancements in the subgap DOS. Insets show the full spectra. 

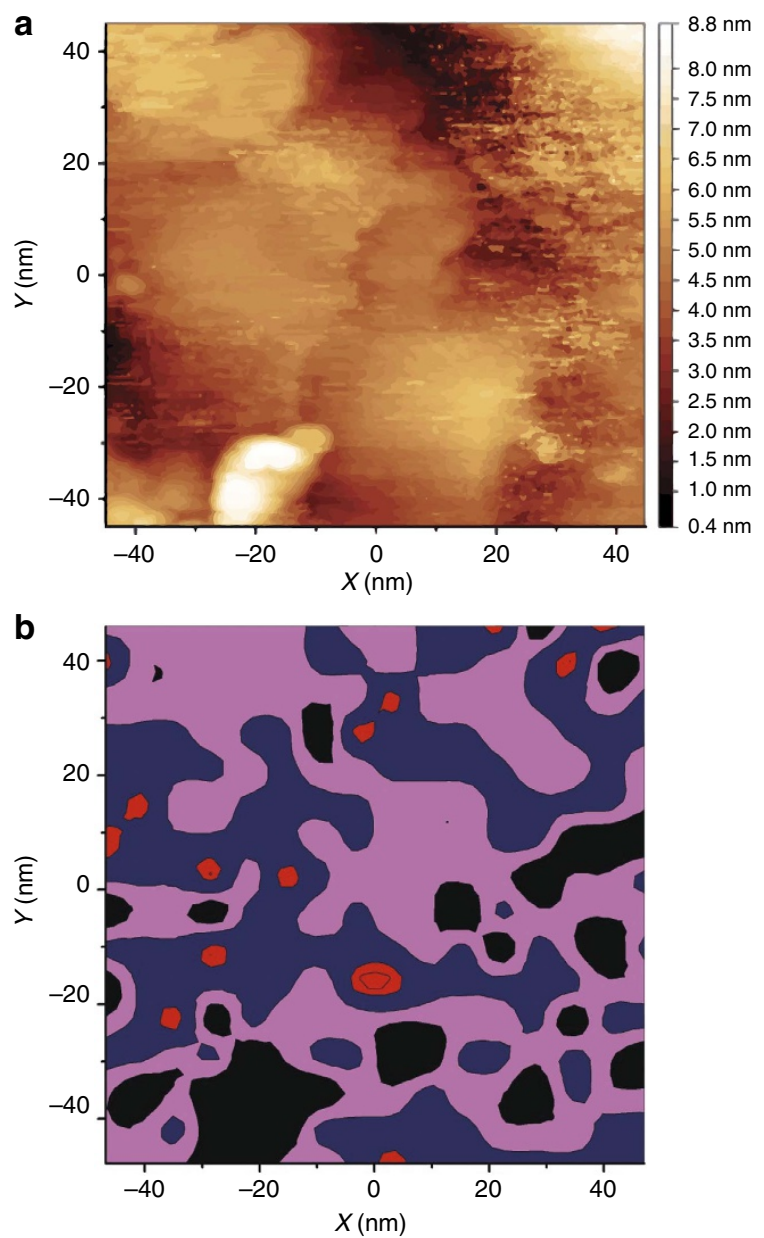
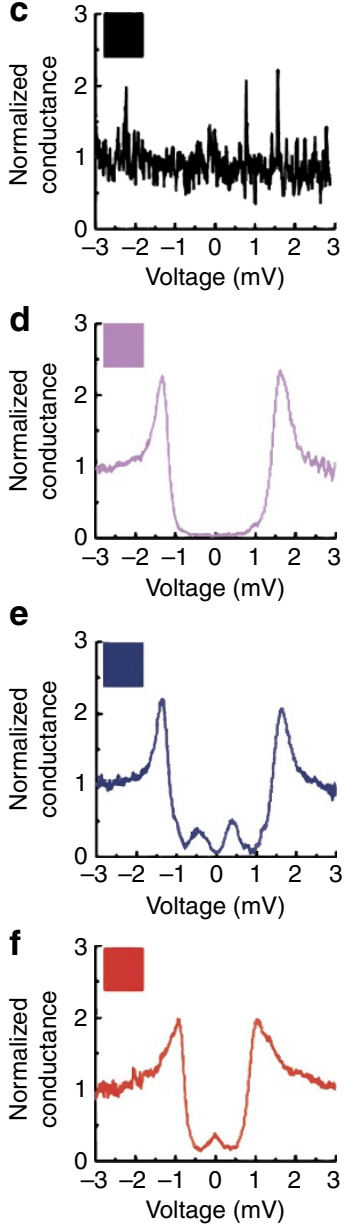

Figure 3 | Spatial dependence of the subgap density of states. (a) Surface topography of the Au (3nm)/Nb (20 nm)/Ho (9.5 nm)/Nb (6.5 nm)/sapphire samples (right key indicates sample height) and in (b) a spatial map of the corresponding spectra obtained at 290 mK; the different colours correspond to the different types of spectra observed and match the spectra shown in $\mathbf{c}-\mathbf{f}$.

fields have on the double peak spectra (similar to that shown in Figs $2 \mathrm{a}$ and 3e). In Fig. 5 we have plotted such spectra in zero field and following the application of successively larger in-plane set fields $H_{\mathrm{SET}}$, which cover the metamagnetic transition of Ho (other fields were also measured but are not shown for clarity). The same field sequence as that used to acquire the magnetic phase diagram in Fig. 1c was applied: (1) spectra were measured with the in-plane field on $\left(H_{\mathrm{SET}}\right)$; (2) the field was switched off and spectra in zero field were obtained; Steps 1 and 2 were repeated with successively larger values of $H_{\text {SET }}$. Spectra with the field on or off show qualitatively similar behaviour: a disappearing subgap structure in the $\mathrm{F}$ phase $(500 \mathrm{mT}$; Fig. 5f); a field-dependent subgap structure below $200 \mathrm{mT}$ where the bulk helix hardly changes with applied field (see Fig. 1c); and the appearance of a zero peak near $150 \mathrm{mT}$.

This behaviour is clearest in Fig. 5g, where we have plotted the difference $(\Delta G)$ in the differential conductance obtained at double peak voltage values to the differential conductance obtained at zero voltage as a function of $H_{\mathrm{SET}}$. Positive values of $\Delta G$ indicate dominant double peaks, while negative values indicate a dominant zero peak. The trend of $\Delta G\left(H_{\mathrm{SET}}\right)$ can be correlated to the magnetic structure of Ho. For fields between 200 and $500 \mathrm{mT}$, the entire magnetic helix in Ho (including surface states) irreversibly deforms and so the degree of magnetic inhomogeneity must decrease causing rapid suppression of the odd frequency components ${ }^{3,4}$, meaning that the DOS should resemble a BCS gap, consistent with the spectra shown in Fig. 5f. The spectra between 0 and $250 \mathrm{mT}$ are perhaps the most interesting since the subgap structure shows an inversion in the sign of $\Delta G$, despite the fact that the bulk helix in Ho is largely unaffected by magnetic fields in this range (Fig. 1c).

In the low field regime $(<250 \mathrm{mT})$, the helix remains intact at least in the bulk of the Ho (Fig. 1c); however, at the surface of Ho the interlayer antiferromagnetic nearest-neighbour exchange-coupling energy abruptly decreases, and the surface stabilizes ferromagnetically (in-plane) over several atomic planes ${ }^{36,37}$. From the $M_{\mathrm{r}} / M_{\mathrm{s}}$ ratio of $\sim 0.08$ at $250 \mathrm{mT}$, we estimate an $\mathrm{F}$ region of $\sim 0.5 \mathrm{~nm}$ per surface or approximately two atomic planes (the $c$ axis parameter at low temperature is $0.564 \mathrm{~nm}$ ). Such a surface is characteristic of a spin-active interface and indeed in this field regime a zero-energy peak appears in the DOS, which is the sought after signature of the odd frequency spin-one triplet component ${ }^{20,21}$.

We stress here that spectra similar to that shown in Fig. 3c,d were unaffected by magnetic field, which is consistent with the view that in those regions with pure BCS gaps or metallic structure the proximity coupling between $\mathrm{Ho}$ and $\mathrm{Nb}$ is weak. We performed additional tests to rule out artefacts because of magnetostrictive drifts. For instance, if a double-peak structure vanished by increasing the magnetic field, we scanned the surrounding area to check whether it appeared in a position nearby but none were observed. The double-peak subgap 

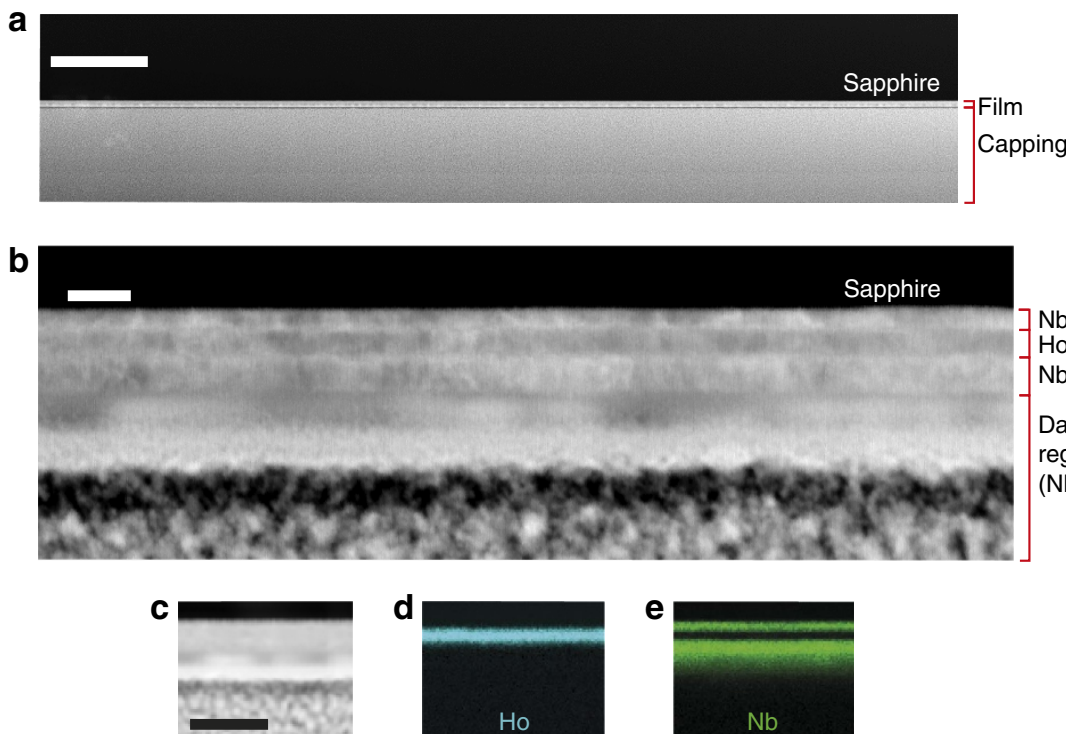

Sapphire

\section{$\mathrm{Nb}(6.5 \mathrm{~nm})$}

Ho $(9.5 \mathrm{~nm})$

$\mathrm{Nb}(12 \mathrm{~nm})$

Damaged

region

(Nb/Au/cap)

f

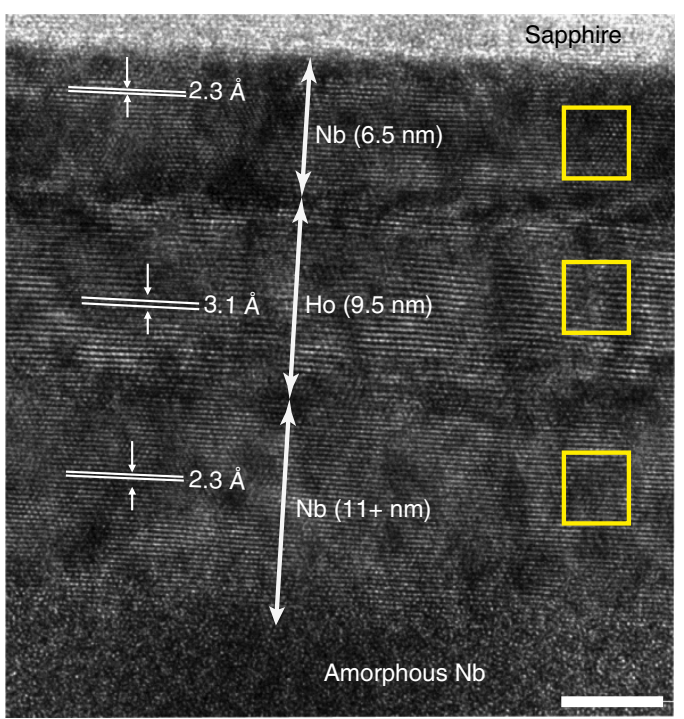

d

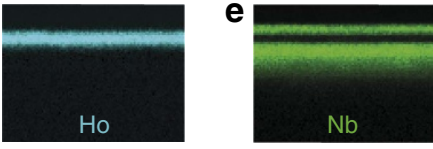

g

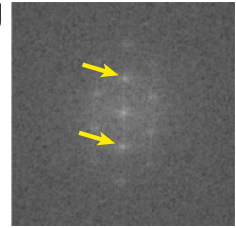

h

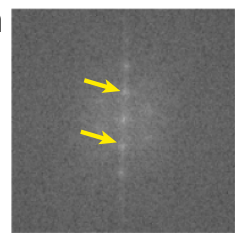

i

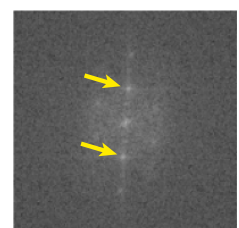

Figure 4 | Electron microscopy of $\mathbf{A u} / \mathbf{N b} / \mathbf{H o} / \mathbf{N b}$ lamellas. (a) Low-magnification scanning transmission electron microscopy (STEM) image showing continuity of the film (scale bar has a length of $1 \mu \mathrm{m}$ ) and (b) a higher-magnification STEM image (scale bar has a length of $20 \mathrm{~nm}$ ). (c) A STEM image and corresponding energy-dispersed X-ray (EDX) maps highlighting $\mathrm{Ho}((\mathbf{d})$ blue) and $\mathrm{Nb}((\mathbf{e})$ green) layers (scale bar in $\mathbf{c}$ has a length of $50 \mathrm{~nm}$; images c-e have matching widths and lengths). (f) A high-resolution transmission electron micrograph of the lamella (scale bar has length of $5 \mathrm{~nm}$ ) and corresponding reciprocal space information (fast Fourier transforms) measured in $\mathrm{Nb}(\mathbf{g}, \mathbf{i})$ and $\mathrm{Ho}(\mathbf{h})$. In $\mathbf{g}, \mathbf{i}$, the arrows indicate reflections from (110) planes, while in $\mathbf{h}$ arrows indicate (002) reflections.

structure of Fig. 5 has been recorded in an area where no zero-bias peak is observed in the as-cooled DOS.

To understand further the behaviour of zero-energy peaks, we investigated the effect of a magnetic field where zero peaks dominate the as-cooled DOS. As shown in Fig. 6, the zero peak first increases with field before disappearing before the onset of the metamagnetic transition. This is consistent with our hypothesis that such zero-energy peaks are related to the F surface of Ho. This may also explain why zero peaks do not always appear in the as-cooled state as the magnetic noncolinearity at the surface of Ho is likely to spatially vary.

The fact that the zero-energy peak in Fig. 6 first increases with field before decreasing is broadly consistent with the zero peak behaviour observed in Fig. 5 (although the initial surface magnetic state of Ho must be different in Fig. 5 than in Fig. 6 as the zero field peak was absent in as-cooled state). The behaviour of the zero peaks in both Figs 5 and 6 suggests that the degree of noncollinearity between the surface $\mathrm{F}$ component and the helix initially increases with field until the field is sufficient to align the surface spins to the top spins of the helix and initiate the metamagnetic transition $(>150 \mathrm{mT})$.

We also investigated the temperature dependence of a zero peak and the effect of out-of-plane magnetic fields to rule out Kondo as an explanation of their appearance. The zero peaks typically disappear below $1 \mathrm{~K}$ (Supplementary Fig. 8), although at higher temperatures the differential conductance becomes noisy since the tip-sample position is unstable. With an out-of-plane field, the magnitude of a zero peak is always found to decrease and none were observed to split with field (Supplementary Fig. 9), which is inconsistent with a Kondo effect. The zero peaks disappear below an out-of-plane field of $500 \mathrm{mT}$ (Supplementary Fig. 9). 
a
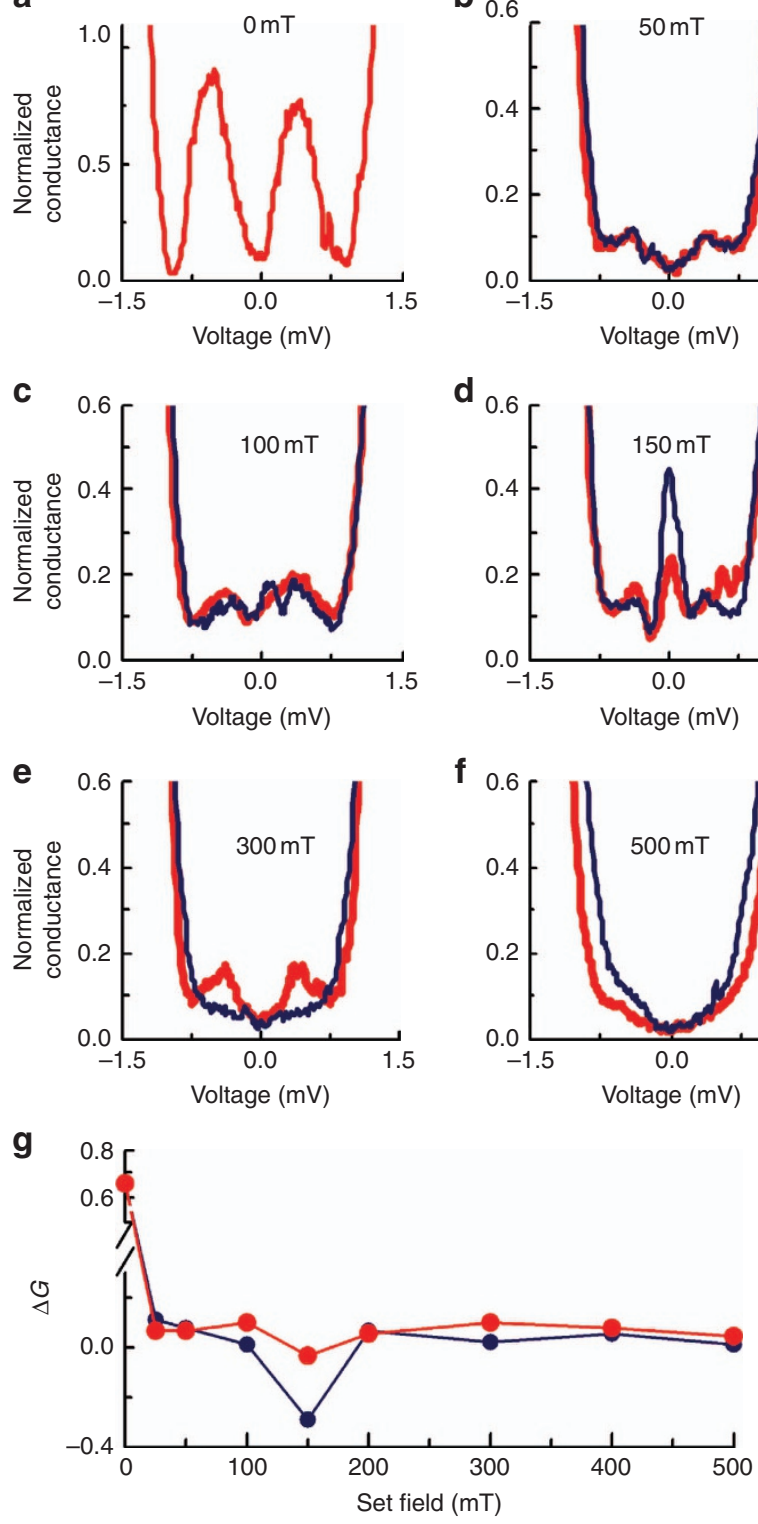

Figure 5 | Effect of magnetic field on the double-peak subgap density of states. (a) Double-peak subgap structure in zero field at $290 \mathrm{mK}$. (b-f) The effect of switching on (blue) and off (red) successively larger in-plane set fields (as labelled) across the metamagnetic transition of Ho. Data were acquired at the same location. $(\mathbf{g})$ The difference $(\Delta G)$ in the differential conductance obtained at side-peak voltages to the differential conductance obtained at zero voltage versus the applied set field $H_{\mathrm{SET}}$ for the field off (red; open circles) and on (blue; solid circle).

\section{Discussion}

The existence of the subgap structure alone indicates unconventional superconductivity; however, to understand more fully the subgap structure and its relation to odd frequency correlations and that of the zero-energy peak to surface magnetism, we have numerically calculated the DOS using the quasiclassical theory of superconductivity in the diffusive limit and solved in a fully self-consistent way (see Supplementary Note 2). This includes both the suppression of superconductivity near the interface region and the induced odd frequency pairing in the superconducting region.

Figure 7 shows the obtained DOS in the superconducting region near the $\mathrm{Au} / \mathrm{Nb}$ interface using the following realistic material parameters: $R_{\mathrm{B}} / R_{\mathrm{S}}=2, R_{\mathrm{S}}=R_{\mathrm{F}}, \xi_{\mathrm{S}}=15 \mathrm{~nm}, h / \Delta=3$, $\lambda=3.4 \mathrm{~nm}, \quad \tau_{F, \varphi}=1.3, \quad \tau_{S, \varphi}=0.4, \quad d_{\mathrm{S}}=20 \mathrm{~nm}, \quad d_{\mathrm{F}}=9.5 \mathrm{~nm}$, $D_{\mathrm{F}} / D_{\mathrm{s}}=0.65$, where $R_{\mathrm{B}}$ is the resistance of the barrier between $\mathrm{Nb}$ and $\mathrm{Ho}, R_{\mathrm{S}(\mathrm{F})}$ is the bulk resistance of the $\mathrm{Nb}(\mathrm{Ho})$ region, $D_{\mathrm{F}(\mathrm{S})}$ is the diffusion coefficient of the Ho $(\mathrm{Nb})$ layer, $\xi_{\mathrm{S}}$ is the superconducting coherence length, $h$ is the exchange field of Ho, $\Delta$ is the superconducting energy gap in $\mathrm{Nb}, \lambda$ is the period of the bulk helix in Ho, $d_{\mathrm{S}}$ is the thickness of the Nb layer, $d_{\mathrm{F}}$ is the thickness of Ho and $\tau_{\mathrm{S}(\mathrm{F}), \varphi}$ describe the spin-dependent interface scattering on the $S(F)$ side of the interface.

In the as-cooled state, we assume that a magnetic helix forms in Ho and we model the effect of surface noncollinearity via spin-active boundary conditions (see Supplementary Note 2). In Fig. 7a there is no misalignment between the surface component and the helix and the double-peak structure dominates, while in Fig. $7 \mathrm{~b}, \mathrm{c}$ the surface component is misaligned $\left(45^{\circ}\right.$ and $\left.90^{\circ}\right)$ with respect to the helix in the basal plane, in which case a dominant zero-energy peak is observed. In the fully $\mathrm{F}$ state, the subgap structure disappears (Fig. 7d).

The experimental features are qualitatively well described by this model, as noncollinearity between the surface F component and the helix dictates whether the double-peak or zero-energy peak structures arise. This model also provides an indirect explanation for why it is more likely to observe the double peaks than a zero peak in the as-cooled DOS. First, the exact magnetic configuration of Ho is likely to vary across the surface of Ho, and, second, that the likelihood of magnetic noncollinearity at the surface is low since ferromagnetic coupling will always prefer a more collinear surface state that favours the double peak structure.

In the model, the subgap structure arises because of odd frequency pairing correlations. The crossover from double peaks to a zero peak occurs in the model as the degree of noncollinearity increases between the surface $\mathrm{F}$ component and the helix. This effect could be related to going from dominant spin-zero odd frequency components in the collinear alignment to a scenario where all triplets, including the spin-one component (relative to the interface moment), contribute for the case with strong
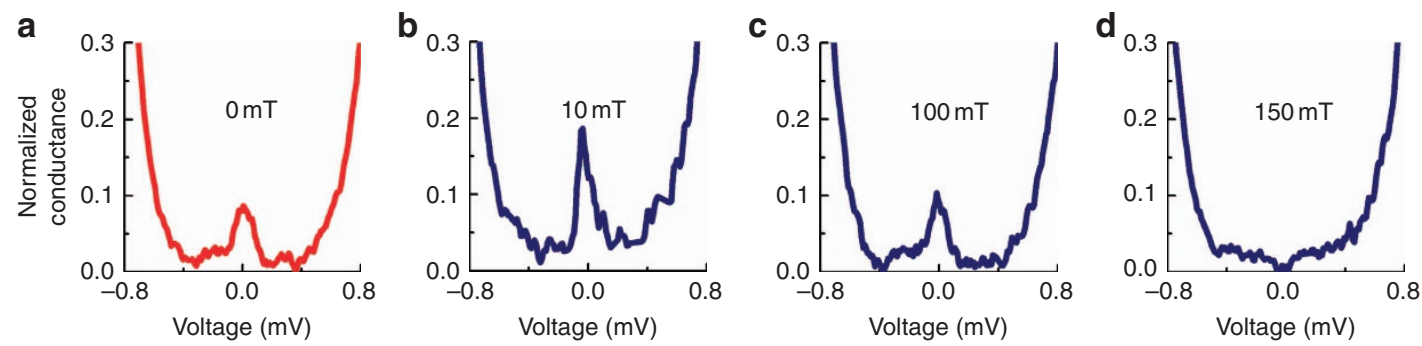

Figure 6 | Effect of magnetic fields on the single-peak subgap structure. (a-d) Zero-peak subgap structure in successively larger in-plane set fields (labelled) at $290 \mathrm{mK}$. Initially, the amplitude of the zero peak increases with applied field (a,b) but then rapidly decreases to zero (150 mT) before the onset of the metamagnetic transition of Ho. 
a

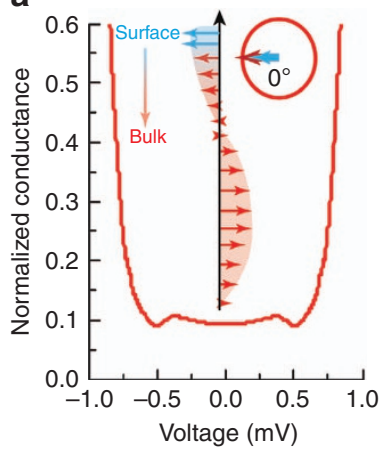

b

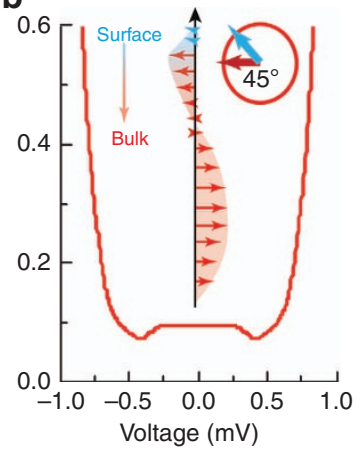

C

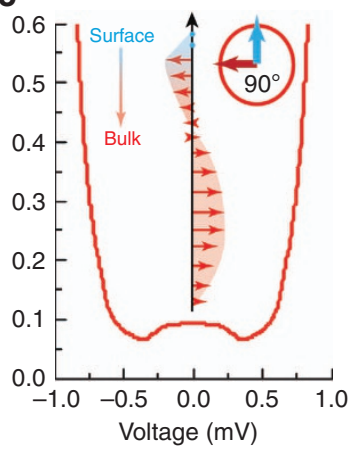

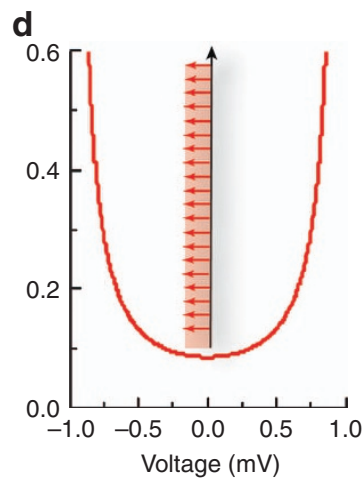

Figure 7 | Calculated magnetic-phase-dependent superconducting DOS in Nb. (a) DOS in Nb for a helical magnetic phase in Ho in which the surface ferromagnetic spins of $\mathrm{Ho}$ (indicated by blue arrows) are aligned or (b) noncollinear by $45^{\circ}$ or (c) by $90^{\circ}$ to the bulk helix in $\mathrm{Ho}$ (indicated by red arrows). (d) DOS in Nb for which the Ho has a ferromagnetic phase as sketched showing no subgap structure.

misalignment. Spin-split peaks inside the subgap region of the superconductor have been shown theoretically to occur in an S/F bilayer when only the spin-zero, odd frequency component is present ${ }^{38}$. This occurs because of spin-dependent scattering at the interface even in the absence of any magnetic inhomogeneity, which induces an effective exchange splitting in the superconductor. On the other hand, the generation of the spin-one odd frequency component is more efficient for the noncollinear alignment between the surface F component and the helix. In this case the zero-energy DOS becomes enhanced because of the gapless nature of odd frequency pairing and the fact that, unlike the spin-zero odd frequency component, the electrons in the spin-one pairs are aligned and affected equally by an effective exchange field induced by the spin-active interface so that they should not give rise to any spin-split structure in the DOS. We note that the triplet state observed here is different from that observed in $\mathrm{Sr}_{2} \mathrm{RuO}_{4}$, which is odd parity but even frequency and requires extreme sample purity ${ }^{39}$.

In conclusion, we have observed a magnetic-phase-dependent superconducting subgap DOS in $\mathrm{Nb}$ proximity coupled to Ho. The results demonstrate a profound modification of the superconducting state because of the presence of odd frequency spin-triplet pair correlations. The existence of triplet states in a superconductor is profoundly interesting in its own right. Such available states mean that within the energy gap of a superconductor a finite net spin polarization exists in the absence of dissipation. One intriguing possibility is that the spin-one triplet states could accommodate a spin accumulation, meaning that in a nonequilibrium $S / F$ device, the $S$ layer could serve as a spin sink or channel for transmitting spin in spintronics ${ }^{40}$.

\section{Methods}

Epitaxial thin-film growth. $\mathrm{Au} / \mathrm{Nb} / \mathrm{Ho} / \mathrm{Nb}$ samples were grown by direct current magnetron sputtering on heated $a$-plane (110) sapphire substrates. The deposition chamber was continuously cooled via a liquid nitrogen jacket, giving a base pressure of $\sim 10^{-8} \mathrm{~Pa}$ with the water partial pressure below $10^{-9} \mathrm{~Pa}$ as confirmed by an in situ residual gas analyser. A base layer of 6.5-nm-thick, nonsuperconducting $\mathrm{Nb}(110)$ was first grown at a substrate temperature of $880^{\circ} \mathrm{C}$ using a deposition rate of $0.035 \mathrm{~nm} \mathrm{~s}^{-1}$. This layer was essential to avoid oxidation of Ho and to initiate epitaxial growth. A 9.5-nm-thick layer of epitaxial Ho (002) was then grown on the $\mathrm{Nb}$ at $650{ }^{\circ} \mathrm{C}$ at a rate of $0.035 \mathrm{~nm} \mathrm{~s}^{-1}$. A 20 -nm-thick layer of superconducting $\mathrm{Nb}$ (110) was then grown on Ho. Finally, substrates were allowed to cool to room temperature in $1.5 \mathrm{~Pa}$ of $\mathrm{Ar}$ and finally were capped with a 3-nm-thick layer of polycrystalline Au. While Au protects the underlying structure from oxidation, it also wets well to $\mathrm{Nb}$ and forms a highly stable intermetallic compound with $\mathrm{Nb}$ (and therefore at the $\mathrm{Nb} / \mathrm{Au}$ interface in our experiment).

Scanning tunnelling microscopy. The DOS spectra were acquired using a custom-built scanning tunnelling microscope (STM) in the spectroscopy mode with an IrPt tip, mounted on a ${ }^{3} \mathrm{He}$ cryostat. The STM consists of one-segmented piezo tube for scanning and coarse approach, using slip and stick motion. In situ superconducting magnets can apply in plane and out-of-plane magnetic fields up to $500 \mathrm{mT}$ and 1 Tesla, respectively. The electronic system has a voltage resolution of roughly $20 \mu \mathrm{V}$ at base temperature $(240 \mathrm{mK})$. In-depth instrument details are described in ref. 35 , and its suitability for studying superconducting proximity effects has been demonstrated in ref. 41.

The topography maps were recorded by slowly scanning in the $x$ direction. The images contain $256 \times 256$ data points. For recording the differential conductance $\mathrm{d} I / \mathrm{d} V$ spectra the scan was stopped, and the feedback loop was switched off at a current of $300 \mathrm{pA}$ and a voltage of $3 \mathrm{mV}$. The spectral map Fig. $3 \mathrm{~b}$ contains $16 \times 16$ spectra. The spectra were acquired using a lock-in amplifier. The direct-currentbias voltage was modulated with an a.c. voltage, with root-mean-square amplitude of $14 \mu \mathrm{V}$ and frequency of $733 \mathrm{~Hz}$. The $\mathrm{d} / / \mathrm{d} V$ was measured using a current-tovoltage converter with a gain of $1 \mathrm{VnA}^{-1}$ and the measuring time per spectrum was typically $\sim 1-2 \mathrm{~min}$. All spectra were acquired at a tunnelling resistance of $10 \mathrm{M} \Omega$ where the signal-to-noise ratio was optimal while avoiding distortions of the spectrum due to the presence of the counterelectrode. This tunnelling resistance is the value that we routinely adopt to perform STM measurements with our set-up.

For higher temperature measurements, the STM was allowed to stabilize for at least $5 \mathrm{~min}$ at each new temperature before spectra were acquired. All spectra are normalized with respect to the ohmic conductance measured at $5 \mathrm{mV}$ and so well beyond the superconducting gap edge and voltage offsets were subtracted using the symmetry of the spectra. To ensure reproducibility of the observed superconducting-related spectral features and minimize noise contribution, each differential conductance spectrum is the average of three scans performed on the same sample spot.

TEM. Lamellae for TEM analysis were prepared using a FEI Helios Nanolab focused ion beam/scanning electron microscope (FIB/SEM). A 2- $\mu \mathrm{m}$-thick capping layer was deposited to protect the film. Scanning transmission electron microscopy-High-Angle Annular Dark Field images and energy-dispersed X-ray maps were acquired on a FEI Osiris (200 kV acceleration voltage) equipped with a high-brightness field emission gun (X-FEG) and a Bruker Super-X detector.

\section{References}

1. Giaever, I. Energy gap in superconductors measured by electron tunneling. Phys. Rev. Lett. 5, 147-150 (1961).

2. Bardeen, J., Cooper, L. \& Schrieffer, J. Microscopic theory of superconductivity. Phys. Rev. 106, 162-164 (1957).

3. Bergeret, F. S., Volkov, A. F. \& Efetov, K. B. Enhancement of the Josephson current by an exchange field in superconductor-ferromagnet structures. Phys. Rev. Lett. 86, 3140-3143 (2001).

4. Bergeret, F. S., Volkov, A. F. \& Efetov, K. B. Odd triplet superconductivity and related phenomena in superconductor-ferromagnet structures. Rev. Mod. Phys. 77, 1321-1373 (2005).

5. Khaire, T. S., Khasawneh, M. A., Mazin, A., Pratt, W. P. \& Birge, N. O. Observation of spin-triplet superconductivity in Co-based junctions. Phys. Rev. Lett. 104, 137002 (2010).

6. Robinson, J. W. A., Witt, J. D. S. \& Blamire, M. G. Controlled injection of spintriplet supercurrents into a strong ferromagnet. Science 329, 59-61 (2010).

7. Klose, C. et al. Optimization of spin-triplet supercurrent in ferromagnetic Josephson junctions. Phys. Rev. Lett. 108, 127002 (2011).

8. Anwar, M. S., Veldhorst, M., Brinkman, A. \& Aarts, J. Long range supercurrents in ferromagnetic $\mathrm{CrO}_{2}$ using a multilayer contact structure. Appl. Phys. Lett. 100, 052602 (2012). 
9. Witt, J. D. S., Robinson, J. W. A. \& Blamire, M. G. Josephson junctions incorporating a conical magnetic holmium interlayer. Phys. Rev. B 85, 184526 (2012).

10. Robinson, J. W. A., Chiodi, F., Egilmez, M., Halász, G. B. \& Blamire, M. G. Supercurrent enhancement in Bloch domain walls. Sci. Rep. 2, 699 (2012).

11. Gingrich, E. C. et al. Spin-triplet supercurrent in $\mathrm{Co} / \mathrm{Ni}$ multilayer Josephson junctions with perpendicular anisotropy. Phys. Rev. B 86, 224506 (2012).

12. Pal, A., Barber, Z. H., Robinson, J. W. A. \& Blamire, M. G. Pure second harmonic current-phase relation in spin filter Josephson junctions. Nat. Commun. 5, 3340 (2014).

13. Leksin, P. V. et al. Evidence for triplet superconductivity in a superconductorferromagnet spin valve. Phys. Rev. Lett. 109, 057005 (2012).

14. Jara, A. A. et al. Angular dependence of superconductivity in superconductor/ spin-valve heterostructures. Phys. Rev. B 89, 184502 (2014).

15. Flokstra, M. G. et al. Controlled suppression of superconductivity by the generation of polarized Cooper pairs in spin valve structures. Phys. Rev. B 91, 060501 (2015).

16. Wang, X. L. et al. Giant triplet proximity effect in superconducting pseudo spin valve with engineered anisotropy. Phys. Rev. B 89, 140508 (R) (2014).

17. Banerjee, N. et al. Evidence for spin-selectivity of triplet pairs in superconducting spin-valves. Nat. Commun. 5, 3048 (2014).

18. Singh, A., Voltan, S., Lahabi, K. \& Aarts, J. Colossal proximity effect in a superconducting triplet spin valve based on the half-metallic ferromagnet CrO. Phys. Rev. X 5, 021019 (2015).

19. Robinson, J. W. A. Viewpoint: a boost for superconducting logic. Physics 8, 49 (2015).

20. Linder, J., Sudbø, A., Yokoyama, T., Grein, R. \& Eschrig, M. Signature of oddfrequency pairing correlations induced by a magnetic interface. Phys. Rev. B 81, $214504(2010)$

21. Yokoyama, T., Tanaka, Y. \& Golubov, A. A. Manifestation of the odd-frequency spin-triplet pairing state in diffusive ferromagnet/superconductor junctions. Phys. Rev. B 75, 134510 (2007).

22. Kontos, T., Aprili, M., Lesueur, J. \& Grison, X. Inhomogeneous superconductivity induced in a ferromagnet by proximity effect. Phys. Rev. Lett. 86, 304-307 (2001).

23. SanGiorgio, P., Reymond, S., Beasley, M. R., Kwon, J. H. \& Char, K. Anomalous double peak structure in superconductor/ferromagnet tunneling density of states. Phys. Rev. Lett. 100, 237002 (2008).

24. Boden, K. M., Pratt, W. P. \& Birge, N. O. Proximity-induced density-of-states oscillations in a superconductor/strong-ferromagnet system. Phys. Rev. B 84, 020510 (R) (2011).

25. Kalcheim, Y., Kirzhner, T., Koren, G. \& Millo, O. Long-ranged proximity effect in $\mathrm{La}_{2 / 3} \mathrm{Ca}_{1 / 3} \mathrm{MnO}_{3} /(100) \mathrm{YBa}_{2} \mathrm{Cu}_{3} \mathrm{O}_{7-\delta}$ ferromagnet/superconductor bilayers: evidence for induced triplet superconductivity in the ferromagnet. Phys. Rev. B 83, 064510 (2011).

26. Kalcheim, Y., Millo, O., Egilmez, M., Robinson, J. W. A. \& Blamire, M. G. Evidence for anisotropic triplet superconductor order parameter in halfmetallic ferromagnetic $\mathrm{La}_{0.7} \mathrm{Ca}_{0.3} \mathrm{MnO}_{3}$ proximity coupled to superconducting $\mathrm{Pr}_{1.85} \mathrm{Ce}_{0.15} \mathrm{CuO}_{4}$. Phys. Rev. B 85, 104504 (2012).

27. Kalcheim, Y. et al. Magnetic field dependence of the proximity-induced triplet superconductivity at ferromagnet/superconductor interfaces. Phys. Rev. B 89, 180506(R) (2014).

28. Buzdin, A. et al. Proximity effects in superconductor-ferromagnet heterostructures. Rev. Mod. Phys. 77, 935-976 (2005).

29. Eschrig, E. \& Löfwander, T. Triplet supercurrents in clean and disordered half-metallic ferromagnets. Nat. Phys. 4, 138-143 (2008).

30. $\mathrm{Gu}$, Y. et al. Magnetic state controllable critical temperature in epitaxial $\mathrm{Ho} / \mathrm{Nb}$ bilayers. APL Mater. 2, 046103 (2014).

31. Koehler, W. C., Cable, J. W., Wilkinson, M. K. \& Wollan, E. O. Magnetic structure of Ho. I. The virgin state. Phys. Rev 151, 414-424 (1966).

32. Koehler, W. C., Cable, J. W., Child, H. R., Wilkinson, M. K. \& Wollan, E. O. Magnetic structure of Ho. II. The magnetization process. Phys. Rev. 158, 450-461 (1967).

33. Strandburg, D. L., Legvold, S. \& Spedding, F. H. Electrical and magnetic properties of Holmium single crystals. Phys. Rev. 127, 2046-2051 (1962).
34. Stöhr, J. \& Siegmann, H. Magnetism: From Fundamentals to Nanoscale Dynamics (Springer, 2006).

35. Debuschewitz, C., Münstermann, F., Kunej, V. \& Scheer, E. A compact and versatile scanning tunneling microscope with high energy resolution for use in a ${ }^{3} \mathrm{He}$ cryostat. J. Low Temp. Phys. 147, 525-535 (2007).

36. Andrianov, A. V. Helical magnetic structures in heavy rare-earth metals as probable manifestation of electronic topological transition. J. Magn. Magn. Mater. 140, 749-750 (1995).

37. Hughes, I. D. et al. Lanthanide contraction and magnetism in the heavy rare earth elements. Nature 446, 650-653 (2007).

38. Cottet, A. Spectroscopy and critical temperature of diffusive superconducting/ ferromagnetic hybrid structures with spin-active interfaces. Phys. Rev. B 76, 224505 (2007).

39. Maeno, Y. et al. Superconductivity in a layered perovskite without copper. Nature 372, 532-534 (2004).

40. Linder, J. \& Robinson, J. W. A. Superconducting spintronics. Nat. Phys. 11, 307-315 (2015).

41. Wolz, M., Debuschewitz, C., Belzig, E. \& Scheer, E. Evidence for attractive pair interaction in diffusive gold films deduced from studies of the superconducting proximity effect with aluminium. Phys. Rev. B 84, 104516 (2011).

\section{Acknowledgements}

The work was funded by the Royal Society through a University Research Fellowship held by J.W.A.R., the Leverhulme Trust (J.W.A.R., E.S. and M.G.B.) through an International Network Grant (IN-2013-033), and the EPSRC through NanoDTC EP/ G037221/1 (A.D.B. and J.W.A.R.). Further support was received from ERC AiG 'Superspin' (M.G.B.). Y.G. acknowledges financial support from King's College, Cambridge. A.D.B. acknowledges additional financial support from the Schiff Foundation and Cost Action MP1201. J.L. was supported by the 'Outstanding Academic Fellows' programme at NTNU and the Norwegian Research Council grants 205591 and 216700. Useful discussion with K. Halterman and F. S. Bergeret are acknowledged. G.D. and C.D. acknowledge funding from the ERC under grant number 259619 PHOTO-EM. C.D. acknowledges financial support from the EU under grant number 312483 ESTEEM2.

\section{Author contributions}

J.W.A.R. had the idea of the experiment and supervised the project. A.D.B. did the STM measurements with the help of S.D. under the supervision of E.S. Y.G. grew the epitaxial films and characterized their magnetic properties under the supervision of M.G.B. The theoretical model was written by J.L. Electron microscopy was performed by G.D. under the supervision of C.D. J.W.A.R. and A.D.B. analysed the measurement data and wrote the paper with contributions from all the other authors.

\section{Additional information}

Supplementary Information accompanies this paper at http://www.nature.com/ naturecommunications

Competing financial interests: The authors declare no competing financial interests.

Reprints and permission information is available online at http://npg.nature.com/ reprintsandpermissions/

How to cite this article: Di Bernardo, A. et al. Signature of magnetic-dependent gapless odd frequency states at superconductor/ferromagnet interfaces. Nat. Commun. 6:8053 doi: $10.1038 /$ ncomms 9053 (2015)

This work is licensed under a Creative Commons Attribution 4.0 International License. The images or other third party material in this article are included in the article's Creative Commons license, unless indicated otherwise in the credit line; if the material is not included under the Creative Commons license, users will need to obtain permission from the license holder to reproduce the material. To view a copy of this license, visit http://creativecommons.org/licenses/by/4.0/ 